\title{
Web 2.0 zwischen technischer und sozialer Innovation: Anschluss an die medientheoretische Debatte
}

\author{
Bastian Pelka und Christoph Kaletka
}

\section{Ein unbestimmter Begriff}

Seit 2004 präsentiert sich das ,Web 2.0’ als neue Entwicklung, die - folgt man jeweils euphorischen oder kritischen Stimmen - nicht mehr und nicht weniger bietet als die endgültige Durchsetzung wahrer Demokratie oder: gar nichts Neues. ${ }^{1}$ Beide Perspektiven haben übrigens nachvollziehbare Argumente; doch dazu später. Fakt ist: Der Begriff des Web 2.0 wurde 2004 vom O’Reilly-Verlag geprägt, der nach dem Platzen der ,dot-com-Blase’ Internet-Experten zu einer Konferenz zur Zukunft des Internet lud. Der Titel der Konferenz - ,Web 2.0 Conference' - war eher dem Marketing der Veranstaltung geschuldet, als einem Ansatz, Ziel oder Konzept zu folgen. Doch er griff: ,Web 2.0' wird heute weltweit als Synonym für eine große Anzahl unterschiedlicher Angebote verwendet und erfreut sich einer regen Diskussion auch in ,klassischen’ Massenmedien und verschiedenen Fachdiskursen.

Dabei leidet der Begriff heute wie 2004 unter einer erstaunlichen inhaltlichen Unbestimmtheit, die sich in einer Vielzahl unterschiedlicher Definitionsversuche bis hin zu dem Ansatz niederschlägt, den Begriff gar nicht zu definieren, sondern als Gesamtheit der ihm zugeordneten Angebote zu verstehen - also als Sammelkategorie. Dieser Ansatz kondensiert in der Tag Cloud ${ }^{2}$, also der grafisch als ,Wolke' dargestellten Assoziation zum Begriff, die zur Zeit die wohl gängigste Definition des Web 2.0 liefert und auch im - dem Web 2.0 zugerechneten - Wikipedia-Eintrag zum Begriff ,Web 2.0’ Verwendung findet. Die Tag Cloud zum Web 2.0 enthält sowohl Angebote (Wikis, Blogs), als auch Gestaltungsprinzipien (Modularity, Joy of Use, Remixability, Simplicity), Technologien (RSS, AJAX) und Nutzungsphänomene (Long Tail, Folksonomies). Die einzige verbindende Klammer dieser Begriffe stellt die Assoziation des Autors

\footnotetext{
${ }^{1}$ Zur Kritik der Genese des Begriffs vgl.: http://de.wikipedia.org/wiki/Web_2.0

${ }^{2}$ Eine Tag Cloud stellt Assoziationen zu einem Begriff dar, indem Assoziationsketten durch Nähe der Begriffe zueinander und die Bedeutung der Begriffe durch deren Größe beschrieben werden.
} 
dar, die jedoch - in der für den gesamten Web 2.0-Diskurs typischen Arbeitsform - durch zahlreiche andere Autoren ergänzt und verändert wurde und damit einen Diskurs repräsentiert.

Abbildung 1: $\quad$ Die hier abgebildete Tag Cloud zeigt die Prinzipien des Web 2.0. Sie wurde von Markus Angermeier am 11. November 2005 veröffentlicht. Quelle: Wikipedia

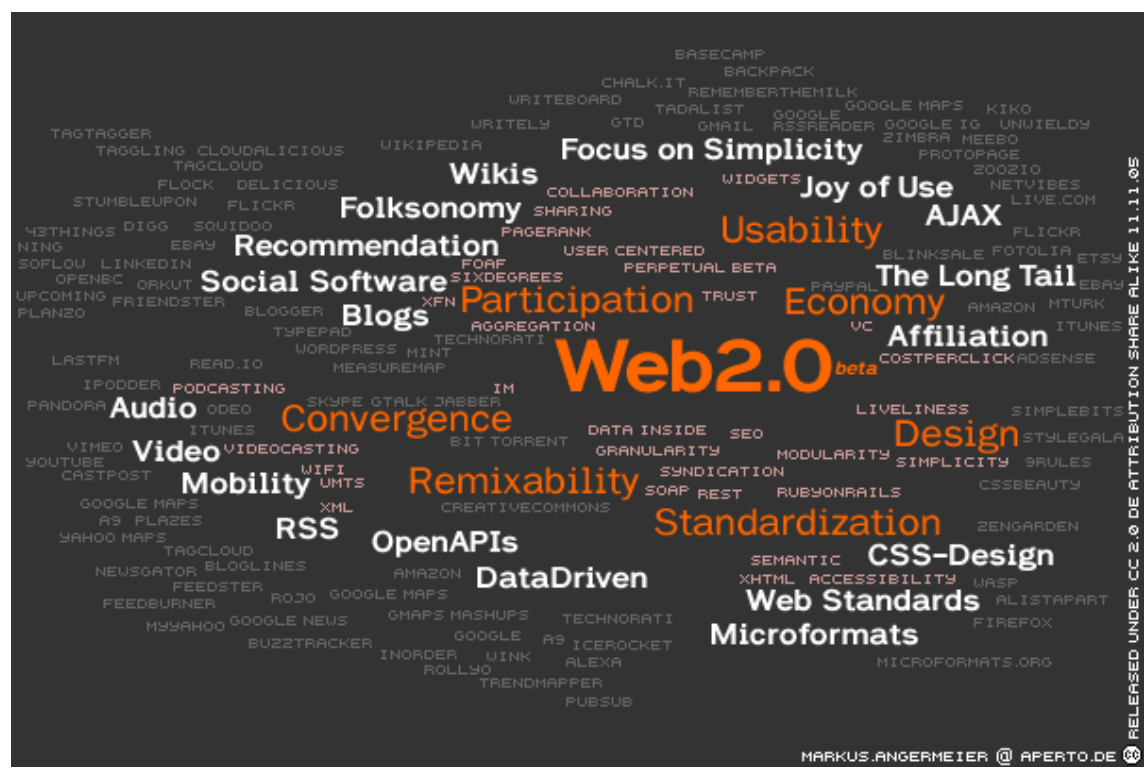

\section{Zwei Innovationsebenen}

Der Begriff ,Web 2.0' weckt aus zwei Gründen das besondere Interesse der Innovationsforschung. Erstens lockt die Breitenwirkung des Begriffes zu einer wissenschaftlichen Auseinandersetzung - immerhin rund 370 Millionen Artikel im Internet beschäftigen sich laut Google mit ihm und 15\% aller deutschen Internet-Nutzer geben an, im Web 2.0 aktiv zu sein; zweitens fordert wohl kaum ein anderer Begriff bereits durch eine implizite Versionierung (,2.0’) so lautstark nach einem Vergleich zum Zustand prae ante und damit der Bennennung seiner Innovation als Differenz.

Aus Perspektive der Innovationsforschung stellt sich jedoch die Frage, ob ein derart undefinierter Gegenstand überhaupt das Objekt ihrer Forschung sein 
darf. Konkret muss Innovationsforschung erklären, wie es gelingen kann, eine Innovation in etwas zu entdecken, das selber nicht definiert ist und somit auch keinen Vergleich zu einem Zustand ohne diese Innovation zulässt. Denn gerade die Vielzahl der im Sammelbegriff vertretenen Bestandteile birgt die stete Gefahr, eine Innovation in etwas zu sehen, das bereits seit langem besteht oder das sich so evolutionär entwickelt hat, das es sich nicht als Entität mit ,Grenzen' fassen lässt. Derjenige, der dies als ,Innovation' bezeichnen wollte, würde also in die Falle tappen, die das Marketing des O’Reilly-Verlags ausgelegt hat.

Nach Einschätzung der Autoren dieses Beitrags kann eine Beantwortung dieser Frage nur über die Erarbeitung einer eigenen, für die Innovationsforschung gültigen, Definition des Web 2.0 führen. Dazu unterscheiden wir zwei Ebenen: eine technologische und eine soziale. Unter der ,technologischen Ebene' soll der Ansatz verstanden werden, im Web 2.0 einen technologischen Versionsschritt zu sehen; dies im Vergleich zu einem - wie auch immer zu definierenden - ,Web 1.0'. Es geht also um die Verbindung des Konzepts ,Web 2.0’ mit einem technologischen Innovationsschritt. Als ,soziale Ebene' wollen wir eine Argumentation entwickeln, die das Web 2.0 als soziale Innovation interpretiert und Neuerungen auf der Ebene der Techniknutzung, nicht der Technik selber, sucht.

Technologische und soziale Innovationen stehen in vielfältigen Verhältnissen zueinander: Soziale Innovation kann, muss sich aber nicht zwingend innovative Techniken zu Nutze machen. Anders herum kann eine innovative soziale Praxis auslösendes Element für technische Innovationen sein und zu deren Verbreitung beitragen (Zapf 1989: 176 ff.). Howaldt/ Kopp/ Schwarz (2008: 64) weisen in diesem Kontext darauf hin, dass Innovation an sich bereits stets einen sozialen Prozess darstellt. Die Bedeutung der sozialen Dimension von Innovationen ist besonders im Kontext der Diskussion von Innovationsfähigkeit thematisiert worden. Denn wirtschaftlicher Erfolg beruht „immer weniger auf territorialen, nationalstaatlich errichteten Barrieren für den freien Personen-, Güter-, Kapital- und Dienstleistungsverkehr und immer mehr auf temporären, durch Innovationen erlangten Vorsprüngen“ (Heidenreich 2004: 125). Das in der LissabonAgenda zum Ausdruck gebrachte gesamteuropäische Innovationsregime (bei aller Differenzierung in angelsächsisch oder kontinentaleuropäisch geprägte nationale Wege) sieht Wertschöpfung durch wissensbasiertes Wachstum, aktive Teilhabe an integrativen Gesellschaften sowie die Schaffung einer wettbewerbsfähigen Wirtschaft als wichtigste Innovationsziele in Europa. Diese Darstellung der Prioritäten der Europäischen Kommission ist aus zwei Gründen interessant: Erstens wird in Reaktion auf die Finanz- und Wirtschaftskrise die Bedeutung und Notwendigkeit sozialer (und sozialstaatlicher) Innovationen hervorgehoben, während technische nur implizit angesprochen werden. Zweitens werden mit Beispielen wie der Teilhabe an integrativen Gesellschaften, der Aneignung neuer 
Fähigkeiten sowie der Förderung von Kreativität und Unternehmergeist genau solche Handlungsfelder genannt, die in einem Resonanzbereich liegen, auf den das Web 2.0 zur Zeit beobachtbaren Einfluss ausübt. Es spricht also vieles dafür, die Ebene der soziales Innovation des Web 2.0 genau zu betrachten. Doch befassen wir uns zunächst mit der Frage, ob es sich beim Web 2.0 nicht doch um eine technologische Innovation handelt.

\subsection{Technologische Ebene}

Die Diskussion um den tatsächlichen Neuerungswert und die Rechfertigung eines einheitlichen Namens (,2.0') für unterschiedliche Angebote wird vor allem im Netz selber, aber auch in techniknahen Fachdiskursen geführt (vgl. statt vieler: Maaß/ Pietsch 2007). Dabei geht es in der Regel um die Frage, ob sich ein ,Versionssprung' von einem ,Web 1.0' zum ,Web 2.0’ beobachten lässt, oder ob die Angebote, die unter der neuen Versionsnummer gelistet werden, nicht vielmehr schon vorher existierten oder durch zahlreiche Evolutionsschritte - auch nach der Namensgebung im Jahr 2004 - entstanden, so dass kein Versionssprung gerechtfertigt sei. Als Grunderkenntnis der Diskussion kann festgehalten werden, dass viele der ,Web 2.0'-Angebote bereits vor der Einführung des Begriffs existierten und die meisten zumindest technisch möglich waren (Maaß/ Pietsch 2007). In der Tat beruhen viele der dem Web 2.0 zugerechneten Angebote auf Software und den im Internet wichtigen ,Standards', die auch 2004 schon lange verfügbar und durchgesetzt waren; jedoch nicht in diesem Umfang angeboten oder nicht genutzt wurden (Rheingold 1992; Bühl 1997). Es lässt sich somit kein Zusammenfallen des ,Entstehungsjahres’ des Begriffs mit einer technologischen Innovation, die einen bedeutenden Einfluss auf Web 2.0-Angebote hat, konstatieren. Oder: „Die Technik und die Programme waren vorher schon vorhanden, nur nicht in einer derart einfachen Weise zusammengefasst.“ (Reißmann 2005: 63) Damit erscheint der Begriff ,Web 2.0’ auf der technologischen Ebene weitgehend arbiträr.

\subsection{Soziale Ebene}

Während auf technologischer Ebene gefragt wurde, ob das Web 2.0 eine neue Technologie darstellt, soll die soziale Ebene die Techniknutzung in den Fokus nehmen. Wir schlagen vor, die technologische Ebene als genotypischen Ansatz und die soziale Ebene als phänotypischen zu verstehen, nach dem sich Web 2.0Angebote durch eine spezifische Nutzungsinnovation auszeichnen. In diesem 
Sinne schließen wir an die Diskussion an, die über den ,Computer als Medium' (Bolz/ Kittler/ Tholen 1994) geführt wurde. Krämer (1998: 11 ff.) erkennt in den Sozialwissenschaften ab spätestens der $90 \mathrm{er} \mathrm{Jahre}^{3}$ - und damit seit der Verbreitung der Computervernetzung - eine zunehmende Bereitschaft, den Computer als (soziales) Medium und nicht nur als (technische) Infrastruktur zu konzipieren. Meist wird der Computer dabei als „Medium interpretiert, das es erlaubt, die zwischenmenschliche Konstellation mündlicher Präsenz für Situationen der Fernkommunikation technisch wiederherzustellen“ (Krämer 1998: 87), also als Mittler von Kommunikation zwischen Menschen. Das Web 2.0 wäre nach diesem Verständnis dann ein Medium, wenn es die Kommunikation zwischen Menschen ermöglicht, wobei der Kommunikationsbegriff an sich jedoch äußerst voraussetzungsreich ist und deutlich das Verständnis eines ,Austauschs von Informationen' übersteigt.

Konkret muss entschieden werden, ob das Web 2.0 über seine bloße Mediationsfunktion als technisches Medium - Wolfgang R. Langenbucher (1985: 272) bezeichnet sie begrifflich treffend als kommunikative Infrastruktur ${ }^{4}-$ hinaus auch als Ort von Informationsproduktion, also als Medium im Sinne sozialfunktionaler Interaktion interpretiert werden kann, wie zahlreiche Ansätze fordern (Kubicek 1997; Weischenberg 1998; Höflich 1995) ${ }^{5}$. Eine nützliche Definition $^{6}$ einer solchen Dichotomie schlägt Herbert Kubicek vor: Er unterscheidet zwischen technischen und sozialen Medien. In Hinblick auf die tatsächliche Verwendung von Medien in größeren sozialen Zusammenhängen stellen technische Medien eine „unfertige Technik“ (Kubicek 1997: 33) dar, weshalb über dieses technische Medienverständnis die ,Medien zweiter Ordnung' gestülpt werden, die eben die soziale Einbettung der Technik in die Kommunikation leisten. Medien zweiter Ordnung erfordern weitere Abstimmungs-, Koordinations- und Regelungsprozesse zwischen den Kommunikationsteilnehmern und kombinieren mehrere Techniken und Funktionen so, dass ein sinnhaft-sozialer Kommunikations- oder Interaktionszusammenhang entstehen kann (Kubicek 1997: 34). Irene Neverla bevorzugt anstatt dieses Funktionsbündels den Begriff ,Vergesellschaftung' und spricht erst dann von Medien, „wenn sie über die Funktion eines technischen Vermittlungssystems hinaus in einen spezifischen institutionalisierten Handlungskontext eingebunden sind“ (1998: 29 f.). Werner

\footnotetext{
${ }^{3}$ Hügli (1997: 122) schon in den 80er Jahren.

${ }^{4}$ So stellt etwa die Druckindustrie die kommunikative Infrastruktur der Printmedien dar.

${ }^{5}$ Die angeführten Ansätze kommen in einer Mediendefinition überein, die eine sozial-funktionale Perspektive beinhaltet.

${ }^{6}$ Eine weitere Perspektive bietet Krämer (1998: 11) an, wobei sie zwischen literarischen, technischen und Massenmedien differenziert. Diese Gliederung erscheint jedoch aufgrund der unterschiedlichen Blickwinkel wenig hilfreich, insbesondere die Trennung zwischen literarischen und Massenmedien kann hier nicht fruchtbar weiterentwickelt werden.
} 
Wirth und Wolfgang Schweiger (1999: 46) prägen für Kubiceks soziale Funktion und Neverlas Vergesellschaftung die eingängige Bezeichnung ,Spielregeln’. Erst durch ,Spielregeln’ avanciert eine Infrastruktur zu einem Medium.

Fassen wir an dieser Stelle zusammen: Alle beschriebenen Ansätze unterscheiden die technische ,Vermittlung' (Mediatisierung im Wortsinn) von ,Medien', die eine sozial-funktionale Einbettung in den gesellschaftlichen Kommunikationsprozess leisten. Damit wird ein zweistufiges Medienverständnis eingeführt, wobei die zweite Stufe eine deutliche soziale Integration des Mediums und dessen Aussagenentstehung aufweist. Im Folgenden soll darum der Frage nachgegangen werden, ob das Web 2.0 ein ,Medium' im Sinne des hier umrissenen Diskurses darstellt.

Wichtig erscheint in diesem Kontext zunächst die Frage der tatsächlichen Nutzung. Betrachtet man die Verbreitung der Internet-Nutzung in Deutschland, kann für die letzten Jahre eine deutliche quantitative Ausweitung der Nutzerzahl festgestellt werden: Waren 1997 noch weniger als 5 Millionen Deutsche online, so hatten 2003 bereits knapp 35 Millionen und 2008 rund 43 Millionen Deutsche Zugriff auf das Internet (ARD/ZDF Onlinestudie 2009). Gleichzeitig veränderten sich die sozio-demographischen Merkmale der Onliner: Von der homogenen Nutzerschicht aus technik-affinen jungen, gut gebildeten Männern hat sich heute die Onliner-Gemeinde in vielen Merkmalen der deutschen Durchschnittsbevölkerung genähert. Damit sind heute bedeutend mehr, aber auch weniger technikerfahrene Menschen ,online'. Diese Nutzererweiterung kann als wichtigeres Element der Genese des Web 2.0 verstanden werden, denn viele Angebote des Web 2.0 funktionieren erst durch eine sehr große Nutzerschaft. Sie ist aber auch konstituierend für die oben gestellte Frage, ob das Web 2.0 als ,Medium' gefasst werden kann, da dieses Konzept eng mit der tatsächlichen Nutzung verbunden ist. Betrachten wir darum drei Beispiele. ${ }^{7}$

\section{Beispiele}

\subsection{Wiki}

Ein $\mathrm{Wiki}^{8}$ ist ein Online-Lexikon, dessen Inhalte von Autoren kooperativ und verteilt erstellt, verändert und diskutiert werden können. Es muss unterschieden werden zwischen der Software, den Inhalten und dem Angebot eines Wiki (Pelka 2008) - eine Differenzierung, die für alle Angebote des Web 2.0 durchhaltbar und insgesamt Frucht bringend ist, wie die weiteren Beispiele zeigen werden.

${ }^{7}$ Als weitere Beispiele für populäre Anwendungen des Web 2.0 seien genannt: PodCasts, Twitter, RSS. Die hier dargestellte Auswahl folgt dem Bekanntheitsgrad der unterschiedlichen Anwendungen. ${ }^{8}$,Wiki' ist das hawaiische Wort für ,schnell'. 
Die ,Software' verwaltet die Inhalte und bietet dem Nutzer Schnittstellen zur Interaktion mit der Datenbank sowie anderen Nutzern an. Mit ihr können Inhalte gesammelt, diskutiert und publiziert werden. Sie selber tritt für den Nutzer nur insofern in Erscheinung, als dass sie als Schnittstelle zu den Inhalten und als Plattform für Interaktion fungiert. Selber programmieren brauchen die Nutzer nicht. Die ,Inhalte' - im Fall eines Lexikons also Artikel, Verlinkungen und Medien, zusätzlich aber Kommunikation in Form von Diskussionssträngen in Foren - werden von den Nutzern erstellt. Eine ,Redaktion' im Sinne einer herkömmlichen Enzyklopädie haben die meisten Wikis nicht; in deren Rolle schlüpfen die Nutzer (Pelka/ Görting/ Schmitt 2008). Der Begriff ,Angebot' meint beides: Das Zusammenspiel von Software und Inhalten; zusätzlich entsteht durch deren Zusammenspiel ein Möglichkeitsraum, der für den Aufbau von Inhalten durch Nutzer essentiell ist. Das Wiki verbindet damit den eigentlichen Wissensbestand (den ,Content'/ die Inhalte) mit Unterstützungsangeboten zur Kooperation und Kommunikation der Autoren zu eben diesen Inhalten. Zudem ermöglicht ein Wiki den Autoren die Verlinkung der Beiträge untereinander, so dass ein Hypertext entsteht, der als Form sozialer Selektionen verstanden werden kann (Pelka 2003: 112 ff.).

Das bemerkenswerteste Phänomen der bekannten Wiki-Anwendung ,Wikipedia' ist sicherlich deren Umfang und Qualität: Die deutschsprachige Wikipedia umfasst rund eine Million Artikel, die englische sogar 3 Millionen (Stand: Januar 2010). Die hohe Nutzungsdichte wird an der Geschwindigkeit deutlich, mit der Vandalismus, also das Löschen oder Zerstören von Artikeln oder volksverhetzende, kriminelle oder rein werbliche Beiträge beseitigt werden. Die Selbsterhaltung des Angebots funktioniert auf Grund der großen Zahl von Nutzern, die Vandalismus melden oder selber beseitigen. Als entscheidendes Merkmal des Erfolgs der Wikipedia lässt sich somit die große Nutzerzahl herausstellen.

\subsection{Community}

Als ,Community’ werden im populären Web 2.0-Diskurs Online-Netzwerke verstanden. Zu den bekanntesten Angeboten zählen beispielsweise die KarriereCommunity ,Xing', das Schul-Forum ,stayfriends' oder die regional orientierte Community ,Lokalisten'. Eher als Anbieter von Multimedia-Dateien sind hingegen Dienste wie ,youtube' oder ,flickr' bekannt, obwohl sie im Prinzip eine Community darstellen, deren Mitglieder multimediale Dateien publizieren, kommentieren und verwalten. Sie alle bieten den Mitgliedern - auf Basis einer nicht weiter in Erscheinung tretenden Software - Möglichkeiten zur Veröffentli- 
chung von Inhalten, zur Suche nach diesen und zur Kommunikation mit anderen Mitgliedern - beispielsweise durch den Einbezug von Foren, Mail, Instant Messaging oder ,klassischen’ Kommunikationswegen (postalische Adressen, Telefonnummern).

EDV-gestützte Netzwerke oder ,Communities' sind keine Erfindung des Web 2.0, sondern lassen sich bereits für die Zeit des nicht-öffentlichen Internet nachweisen (Rheingold 1992). Dery (1996: 29 ff.) sieht selbst diese frühen Communities als bloße (technische) Fortführung der (auf der sozialen Ebene innovativen) Diskussionskultur der 1960er Jahre und wirft damit die bereits von Howaldt/ Schwarz in diesem Band diskutierte Frage nach dem Verhältnis von technologischer und sozialer Innovation auf (vgl. auch Howaldt/ Schwarz 2009). Hier stellt sich also in besonders dringender Form die Frage nach einer technologischen Innovation von Communities im Web 2.0. Neu an den dem Web 2.0 zugerechneten Angeboten ist jedoch nach Ansicht der Autoren dreierlei: Die Einfachheit, mit der Inhalte - wie z. B. persönliche Websites - angelegt, verwaltet und publiziert werden, die quantitative Reichweite der Web 2.0-communities, die nicht mehr Treffpunkte einer EDV-affinen Elite sind, sondern eine breite Masse von Menschen erreichen, deren primäres Interesse nicht in der EDV selber liegt, und die qualitative Bedeutung der Netzwerke für die Nutzer im Sinne von sozialem Prestige. Es entsteht ein Netzwerk von Mitgliedern, die eine Beziehung untereinander aufbauen, da sie gemeinsame Interessen vertreten und Informationen zu ihren Interessen suchen und veröffentlichen. Oder, mit Wassermann/ Faust: „A social network consists of a finite set of actors and the relation or the relations defined on them. The presence of relational information is a critical and defining feature of a social network.” (Wasserman/ Faust 1994: 20)

\subsection{Blog}

Ein Blog - ein Kunstwort aus den Begriffen ,Web’ und ,Log', also ,Internet' und ,Logbuch' - ist ein Tagebuch im Internet. Der Suchdienst ,Technorati’ zählt weltweit über 80 Millionen Blogs, diese weisen jedoch eine stark heterogene Qualität auf. So schätzt Pohlmann (2007), dass nur rund 16 Millionen wirklich aktiv sind. Ein Blog wird von einem ,Blogger' auf einer Website im Internet geführt und richtet sich damit prinzipiell an alle Nutzer des Internet. Es gibt nur wenige gestaltende Prinzipien von Blogs, da das wichtigste Merkmal ihre technische Einfachheit ist (,Simplicity'). Das Blog besteht aus einer Startseite, auf der der jeweils neueste Eintrag (auch ,post' oder ,posting' genannt) oben steht und ältere nach unten verschiebt - das Blog ist also prinzipiell unendlich lang und stellt durch den Einbezug von Verlinkungen und Suchfunktionen ein Archiv dar. 
Ein Eintrag besteht aus Überschrift und Anreißer sowie einem Link auf den vollständigen Text. Vielfach stellt der Anreißer keine eigene textliche Leistung dar, sondern wird lediglich automatisch aus den ersten Zeilen des Beitrags gebildet. Damit verzichtet ein Blog auf die Funktionen, die der Anreißer zum Beispiel den Lesern einer Tageszeitung bietet: Dort steht der Anreißer als - von der Redaktion als ,trainierter' Selektionsinstanz ausgewählter und damit priorisierter - Einstieg in einen Beitrag (Weischenberg 1990: 59 ff.). Genau dieser institutionalisierten Selektion und damit der Übergabe von Entscheidungskompetenz an eine Instanz (im Beispiel der Tageszeitung: die Redaktion) widersetzt sich jedoch ein Blog, indem es auf redaktionelle Standards weitgehend verzichtet.

Da jeder Eintrag eine eindeutige nicht mehr veränderbare Adresse besitzt, können Betreiber anderer Blogs auf diese verweisen - diese Verweise werden ,permalinks' genannt. Durch die Verlinkung zahlreicher Blogs untereinander entsteht ein Bezugsnetzwerk, die ,Blogosphäre'. Über die schnelle und einfache Verbindung verschiedener Blogs sind deren Autoren in der Lage, Nachrichten schnell einer großen Nutzerschaft zur Verfügung zu stellen.

Auch hier muss zwischen den Ebenen der Software, der Inhalte und des Angebots unterschieden werden: Die Software stellt die Schnittstelle zwischen Nutzer und Inhalten bereit und ermöglicht das Publizieren und Verwalten der Inhalte. Blog-Software bringt das Prinzip der Erhöhung der Nutzerschaft durch technische Einfachheit auf den Punkt: Sie lässt sich mittlerweile als Plug-In zum Office-Paket installieren, so dass jeder ein Blog führen kann, der Texte in Word tippen kann.

Die bekannteste Studie zur Untersuchung von Blogs im Vergleich zu ,klassischen' Medien und damit zur Innovation, die Blogs darstellen, legen die Kommunikationswissenschaftler Neuberger/ Nuernbergk/ Rischke (2007) vor. Sie konstatieren durch Blogs einen „Übergang vom redaktionell gesteuerten Publizieren zur Partizipation“ (Neuberger/ Nuernbergk/ Rischke 2007: 96). Von besonderer Bedeutung erscheint insbesondere die Kraft von Blogs zum medialen Agenda Setting, also der Beeinflussung von Themen, die durch Medien aufgegriffen werden. Ein eindrucksvolles Beispiel für das Agenda Setting einer Web 2.0-gestützten sozialen Bewegung liefert die ,San Precario'-Bewegung in Italien und Spanien. Politische Aktivisten erfanden dort ,San Precario' als Schutzheiligen der prekär Beschäftigten und publizierten ihn über zahlreiche Blogs. Die Schnelligkeit, Einfachheit und die geringen Kosten dieser Kommunikationsform unterstützten das Agenda Setting des Schutzheiligen, so dass mittlerweile ein europaweites Netz von Aktivisten entstanden ist, das auch in klassischen Massenmedien stark wahrgenommen wird. 
Klassische Medien nahmen diese Themen auf, liefern in einem reflexiven Prozess aber auch Themen, die in Blogs diskutiert werden (Leskovec/ Backstrom/ Kleinberg 2009).

\section{Definition des Web 2.0 als soziale Innovation}

Kehren wir nach diesen drei Beispielen zur Frage der Innovationsebene des Web 2.0 zurück. Nachdem auf technologischer Ebene ein Innovationsschritt verneint wurde, sollten durch die Nutzungsbeispiele Innovationen auf der sozialen Ebene untersucht werden. Es wurde deutlich, dass das Web 2.0 eine neue Nutzungsweise ,alter Technologien' darstellt, die jedoch eine (quantitativ wie qualitativ) breitere Nutzerzahl (und damit Internet-Zugänge) benötigt. Oder, mit Alby (2007: 11): „Die Systemanforderungen an das Web 2.0 waren der Nutzer 2.0, der selbst Zugangsgeschwindigkeit 2.0 und Zugangskosten 2.0 erforderte.“

Die Beispiele haben gezeigt, dass die Angebote des Web 2.0 auf die aktive Einbeziehung einer breiten und vernetzten Nutzerzahl angewiesen sind. Damit kann nun auch die oben aufgeworfene Frage beantwortet werden, ob es sich bei Angeboten des Web 2.0 um ,Medien' im dort skizzierten Verständnis handelt. Da die untersuchten Angebote die von Kubicek (1997) geforderten Abstimmungs-, Koordinations- und Regelungsprozesse geradezu zum Erkennungsmerkmal erheben, können diese als ,Medien zweiter Ordnung' gefasst werden. Sie zeichnen sich gerade durch sinnhaft-soziale Kommunikationszusammenhänge (Kubicek 1997: 34) zwischen den Nutzern aus.

Zwar können auch für die Vernetzung von Nutzern Beispiele aus der Zeit vor der Entstehung des Begriffs ,Web 2.0' angeführt werden; so beschreibt Achim Bühl (1997) bereits gesellschaftliche Veränderungen durch den Einsatz vernetzter Computer. Symptomatisch erscheint jedoch, dass er in seiner Aufzählung der „realisierten Anwendungen“ (Bühl 1997: 167 ff.) überwiegend Nischenoder Spitzenanwendungen beschreibt, nicht jedoch die Einbeziehung einer breiten Nutzerschaft.

Die Innovation des Web 2.0 liegt somit - das haben die drei Beispiele gezeigt - im Ansatz des ,user generated content'- also der Übergabe der Erstellung der veröffentlichten Inhalte an eine breite Nutzerschicht und damit der sozialfunktionalen Nutzung der Infrastruktur. Denn im Vergleich zu ,klassischen’ Medien, bei denen eine Redaktion ${ }^{9}$ Inhalte erstellt und distribuiert, werden alle

${ }^{9}$ Der Blick auf Redaktionen als Orte der Aussageentstehung ist hier extrem verkürzt wiedergegeben. Auch die in der Kommunikationswissenschaft übliche Trennung zwischen Medien und Massenmedien wurde hier ignoriert. Zum Einstieg in die Diskussion: Neuberger/ Nuernbergk/ Rischke 2007; Weischenberg 1998. 
Inhalte bekannter Web 2.0-Angebote von den Nutzern erzeugt und von einer ,Redaktion' des Anbieters lediglich überwacht oder moderiert.

Vor diesem Hintergrund soll das Web 2.0 im Folgenden als ein spezifischer Nutzungskontext des Internet - als Medium erster Ordnung - beschrieben werden, das auf der technischen Innovation des Mediums aufbaut, diese jedoch durch eine soziale Nutzungsdimension erweitert. Kurz: Das Web 2.0 umfasst Angebote (Medien), die über die technische Infrastruktur des Internet genutzt werden und damit der technischen Ebene eine soziale hinzufügen. Das Web 2.0 beschreibt damit eine veränderte Wahrnehmung und vor allem Nutzung des Internet.

Ein Weiteres vereint die verschiedenen Angebote des Web 2.0: Der Nutzer ist gleichzeitig Autor, Vermittler und Kommunikator und kann sich selbstgesteuert Wissen aus dem Angebot aneignen oder gleichzeitig sein Wissen über das Angebot der Öffentlichkeit zugänglich machen und somit selbst zum Wissensanbieter avancieren. Dies führt zur Unterscheidung von zwei Formen von Interaktivität: die Interaktion von Menschen mit einer Technologie (Software) und die Interaktion von Menschen untereinander. Quiring und Schweiger (2006: 21) gelangen nach einer Analyse des Diskurses zum Interaktionsbegriff zu dem Schluss, dass es sich bei der Erklärung von Interaktivität „um ein überdefiniertes Konzept handelt, dem es allerdings sowohl an begrifflicher Klarheit als auch an ausreichenden Systematisierungsbefunden mangelt“. Grund dafür sind die unterschiedlichen Bedeutungen des Begriffs in der Soziologie und Informatik. In der Soziologie beschreibt er „wechselseitig aufeinander bezogene menschliche Handlungen (...), also die Beziehungen zwischen zwei oder mehreren Menschen“ (Goertz 1995: 478). In der Informatik steht jedoch die Kommunikation zwischen Mensch und Maschine im Mittelpunkt der Forschung (Quiring/ Schweiger 2006). Nach Haack (1995: 151) beschreibt Interaktivität „die Eigenschaft von Software, dem Benutzer ein Reihe von Eingriffs- und Steuerungsmöglichkeiten zu eröffnen.“ Darüber hinaus bezeichnet Interaktivität nach (Back/ Bendel/ Stoller-Shai 2001: 296) das Verhältnis zwischen Benutzer und Medium, „so dass man von einer Wechselwirkung zwischen Benutzer und Medium sprechen kann, oder auch davon, dass das Medium selbst interaktiv ist, also eine solche Wechselwirkung zulässt“. Verbunden hiermit ist die Übergabe von Kompetenzen (z. B. der Auswahl des eigenen Lernpfades) an eine Software. Diese Übergabe setzt ein starkes Vertrauen des Lernenden gegenüber der Software voraus (vgl. Kuhlen 1999) und wird durchaus kritisch diskutiert (Pelka 2003: 144 ff.). Für unser Verständnis des Web 2.0 als soziale Innovation sollen beide Ansätze - der ,soziologische' und der ,technische' - nebeneinander gesetzt, aber nicht verschmolzen werden. Es soll zwischen der Interaktion von Mensch und Maschine beziehungsweise Mensch und Mensch unterschieden werden. Applika- 
tionen des ,Web 2.0' wird unterstellt, beide Formen anzubieten: das freie Bewegen in Wissensräumen, wie auch die Kommunikation mit anderen Menschen. ${ }^{10}$

Dieser Anforderung wollen wir mit folgender Definition gerecht werden: Unter ,Web 2.0' sollen interaktive und kollaborative Angebote im Internet verstanden werden, die den Nutzer bei der Erstellung von Inhalten einbeziehen und somit die Erstellung von ,user generated content' sowie die Kommunikation mit anderen Nutzern ermöglichen und fördern. Dabei ist unser ,Content'-Begriff ein weiter. Er umfasst jede Form von Inhalteproduktion, Kommunikation und Partizipation, so zum Beispiel auch die von Tapscott/ Williams beschriebenen ökonomischen Partizipationsprozesse und ,Peer Production' (2007: 11). Diese Nutzerzentrierung zwingt zu einer leicht erlernbaren und intuitiven Bedienbarkeit, da jede technische Hürde die Zielgruppe der möglichen Nutzer verringern würde. Aus dem Anspruch der Technologie, dem Nutzer die Bereitstellung und Bearbeitung der Angebote zuzuweisen, leitet sich somit der Zwang zu einer nutzerfreundlichen Form ab.

Die hier entworfene Definition umfasst alle in der oben dargestellten Tag Cloud genannten ,Anwendungen' (im hier definierten Sinn), jedoch keine der dort genannten Technologien, Gestaltungsprinzipien oder Nutzungsphänomene. Unsere Definition ist also enger als die verbreitete und populäre Tag Cloud. Gleichzeitig ist sie in Bezug auf eine Anwendung auch ,breiter', denn nach ihr fallen auch Foren unter den Begriff ,Web 2.0', obwohl sie eine der ältesten Anwendungen im Internet darstellen. Hiermit wird unsere Schwerpunktsetzung deutlich, die das Web 2.0 als innovative Nutzungsform bestehender Technologien konzeptioniert.

Es gilt noch eine weitere Abgrenzung vorzunehmen: Zwar ist in der Innovationsforschung der Begriff der user innovation eingeführt; er bezieht sich aber eher auf eine unternehmensgesteuerte Beteiligung von Nutzern an der Verbesserung von Produkten (von Hippel 2005; Flowers/ Sinozic/ Patel 2009), nicht auf die völlig freie Erstellung von Content durch Nutzer. Der Unterschied ist gravierend, da die user innovation im Kontext von Unternehmen deutlich mehr Möglichkeiten der Steuerung und Kontrolle von Innovationen unterliegt, wohingegen der Content des Web 2.0 kaum zu kontrollieren ist.

Angebote des Web 2.0 funktionieren nur durch die Beteiligung der Nutzer, sind an sich also inhaltslos. Dies legt die Idee eines Verständnisses des Web 2.0 als ,Raum’ nahe: Der Raum stellt Möglichkeiten zur Verfügung, die jedoch erst durch seine ,Bewohner' realisiert werden.

${ }^{10}$ Der Grad beider Interaktionsdimensionen kann dabei stark variieren. So bieten Beispielsweise communities wie Xing zahlreiche Möglichkeiten frei gestaltbarer Kommunikation (z. B. Chat, Mail, Foren), andere wie etwa youtube lediglich Interaktion durch Kommentierung von publizierten Inhalten (Videos, Töne). 
Eine weitere Analogie lässt sich zum Einzug des world wide web oder des desktop publishing ziehen: Auch bei der Nutzung des Internet-Protokolls wie bei der Erstellung von Druckvorlagen am Computer ermöglichten jeweils Technologien, die dem Nutzer die Erarbeitung des technischen Hintergrundwissens abnahmen, eine enorme Verbreiterung der aktiven Nutzerschaft und damit den Durchbruch als überall verfügbare Technologie. Heute ist die Nutzung des Internet ohne die grafische Oberfläche world wide web oder Vorlagenerstellung ohne desktop publishing kaum mehr denkbar - die Nutzererweiterung hat also die Nutzung selbst verändert.

\section{Web 2.0: ein unbequemer Gegenstand der Innovationsdebatte}

Führt man sich die Engführungen der Definitionen von Web 2.0 bis hierher vor Augen, besteht eigentlich kein Zweifel daran, dass Web $2.0 \mathrm{zu}$ den sozialen Innovationen gerechnet werden muss. Oder? Passt Web 2.0 wirklich in die Reihe von Beispielen, die in diesem Band ausführlich als soziale Innovationen eingeführt werden? Aus kulturökonomischer Perspektive hat Groys Innovation als etwas Neues, das nicht bloß etwas Anderes im Sinne einer Veränderung ist, sondern „das wertvolle Andere“ (Groys 1999) bezeichnet. Innovationen kommt in modernen Gesellschaften eine zentrale Rolle zu, ebenso allerdings der Frage, wie über Innovationen kommuniziert wird. Innovationen, Innovationsfähigkeit und die Übernahme von Innovationen sind Schlüsselgrößen für die Position von Unternehmen innerhalb des Wirtschaftssystems, von Nationen innerhalb der Staatengemeinschaft und für gesellschaftliche Gruppen. Howaldt/ Schwarz stellen in diesem Band fest (vgl. auch 2009: 3), dass es sich dann um eine soziale Innovation handelt, „wenn sie - marktvermittelt oder ,non- bzw. without-profit' - sozial akzeptiert wird und breit in die Gesellschaft bzw. bestimmte gesellschaftliche Teilbereiche diffundiert, dabei kontextabhängig transformiert und schließlich als neue soziale Praktiken institutionalisiert bzw. zur Routine wird“.

Gerade beim Thema Web 2.0 fällt auf, in wie vielen gesellschaftlichen Teilsystemen diese noch junge Innovation als relevant angesehen wird und sich in rasanter Art und Weise durchgesetzt hat. Der Grad der Verbreitung ist hoch, Web 2.0 hat sich aus der Kommunikationsnische computeraffiner ,early adopter' befreit und findet in einer Vielzahl gesellschaftlicher Zusammenhänge alltägliche Anwendung. Warum ist das so? Ein Sprung zurück an den Anfang dieses Artikels: Die Tag Cloud der Assoziationen zu Web 2.0 stellt den Gegenstand weniger als klar definierbaren Begriff denn als Sammelkategorie vor, die neben konkreten Angeboten und Nutzungsformen auch Gestaltungsprinzipien, zugrunde liegende Technologien und spezifische Nutzungsphänomene enthält. Hieraus 
ergeben sich fast zwangsläufig unterschiedliche Auffassungen zum Innovationscharakter, je nachdem auf welche Bereiche der Sammelkategorie man sich bezieht. Unbequem wird die Einordnung von Web 2.0 als Innovation außerdem dadurch, dass allein durch die Betitelung ein klarer Bruch, nicht nur eine graduelle Veränderung der Netznutzung, sondern eine Neuordnung suggeriert wird. Auf technologischer Ebene ist dies, wie gezeigt wurde, nicht haltbar. Ihre Wurzeln haben die Technologien im ,Web 1.0'. Wenn das so ist, worin besteht dann ,das wertvolle Andere', das das Web 2.0 zu einer in sozialen Praktiken institutionalisierten Innovation macht? Hierzu fallen zunächst zwei praktisch nutzbare Merkmale des Web 2.0 auf:

Neue Diffusions- und Disseminationswege: Die für die Nutzung von Web 2.0 grundlegenden, aber schon älteren technischen Mindestlösungen werden ebenso vermarktet wie die Gestaltung und Pflege von Blogs und Wikis, wie Web 2.0 basierte PR-Kampagnen und natürlich Qualifizierungen neuer Nutzerinnen und Nutzer. Diffusion findet primär dadurch statt, dass immer weitere Gruppen als Autoren und Kommentatoren gewonnen werden und die so entstehenden Produkte gleichzeitig zunehmend rezipiert und sozial akzeptiert werden. Web 2.0 als soziale Innovation wird also weniger über technologische Infrastruktur als über den Markt, über soziale Netzwerke und soziale Bewegungen, die als eine der ersten gesellschaftlichen Gruppen kontinuierlich und professionell Web 2.0Kommunikation genutzt haben, verbreitet.

Gesellschaftliche Akzeptanz und gesellschaftlicher Nutzen: Jenseits steigender Nutzerzahlen etablierter Angebote wie twitter, stayfriends oder Wikipedia ist Web 2.0 mittlerweile auch Gegenstand staatlicher Förderung und von ,capacitybuilding’. Ein Beispiel: ,Telecenters' und Telecenter Netzwerke ${ }^{11}$ qualifizieren und fördern die Beschäftigungsfähigkeit benachteiligter Gruppen, bauen - öffentlich gefördert - Online Communities auf, deren Mitglieder sonst keinen Zugang zu internetgestützter Kommunikation hätten, und führen diese durch Nutzung von Wiki gestützten Curricula an die Arbeitswelt heran (Kluzer/ Rissola 2009). Web 2.0 ermöglicht dadurch Gruppen von Menschen, die aus Arbeitswelt und sozialem Leben ausgeschlossen sind, eine neue niedrig schwellige Form kooperativen, partizipativen Lernens und Arbeitens.

Für Deutschland verweist bspw. die Studie „Zukunft und Zukunftsfähigkeit der deutschen Informations- und Kommunikationsbranche“ (Münchener Kreis/ Deutsche Telekom AG/ TNS Infratest GmbH/ European Center for Information and Communication Technologies (EICT) GmbH 2008) auf soziale Nutzungsansätze des Web 2.0, insbesondere zur Überwindung der digitalen Gräben in der Gesellschaft.

${ }^{11}$ Vgl. www.telecentre-europe.org. 
„Dass der Überwindung der ,Digitalen Spaltung’ in Deutschland nicht eine unzureichende technische Verfügbarkeit von breitbandigen Internetzugängen, ökonomische Barrieren oder eine technologiefeindliche Gesellschaft entgegenstehen, bestätigt die vorliegende Expertenbefragung deutlich. Positive Treiber und Hebel, die eine weitere Diffusion der Internetnutzung in Deutschland fördern, liegen zuallererst im Bereich der Bildung.“ (Münchener Kreis/ Deutsche Telekom AG/ TNS Infratest $\mathrm{GmbH} /$ European Center for Information and Communication Technologies (EICT) GmbH 2008: 12)

Gleichzeitig wird der Bedeutung sozialer Innovationen - insbesondere im Hinblick auf die Web 2.0 Nutzung - ein eigenes Kapitel gewidmet. Damit verorten die Herausgeber in den ,Web 2.0'-Technologien ein Potenzial zur Schließung der ,digitalen Spaltung' und setzen damit bemerkenswerterweise eine Technologie gegen Technologieunkenntnis. Was auf den ersten Blick widersinnig erscheint, ergibt vor dem Hintergrund der Ergebnisse von Kluzer/ Rissola (2009) durchaus Sinn: Wenn es gelingt, die technischen Zugangshürden zum Web 2.0 etwa durch pädagogisches Personal in Telecentern - zu senken, eignen sich Angebote des ,Web 2.0' zur selbständigen Nutzung auch durch technikunerfahrene Menschen.

\section{$6 \quad$ Fazit und Ausblick}

Web 2.0 wurde hier als die Gesamtheit der interaktiven und kollaborativen Angebote definiert, die sich durch leicht erlernbare und intuitive Bedienbarkeit auszeichnen und Nutzern ohne nennenswerte technologische Hürden die Erstellung, Wartung und Veröffentlichung von ,user generated content' ermöglichen. Nimmt man diese neue kommunikative Praxis gemeinsam mit dem hohen Verbreitungsgrad in unterschiedlichen gesellschaftlichen Bereichen in den Blick, wird deutlich, dass sich eine „neue soziale Praxis zur Erhöhung der Wirksamkeit technisch vermittelter Kommunikation“ (Zapf 1989: 33) entwickelt hat. Durch die Loslösung der Diskussion von ihrem ,technischen Ballast' wird so auch der Blick frei auf neue Forschungsfragen nach der zukünftigen alltäglichen Durchdringung von Bildung, Erwerbsarbeit, Freizeitverhalten, kulturellen und politischen Partizipation und weiteren Lebensbereichen mit Web 2.0. Anschlussforschung erscheint zu vielen Fragen lohnenswert. So ließen sich die skizzierten Vorteile für Forschungs- (Tapscott/ Williams 2007), Innovations- (Beerheide/ Howaldt/ Kopp 2009 sowie Howaldt/ Kopp/ Schwarz 2008), Wissensmanagement- und Bildungsprozesse fruchtbar machen, aber auch für politische Bewegungen oder das ,Agenda Setting' von Themen, die die traditionellen Medien 
nicht - oder zu spät (Leskovec/ Backstrom/ Kleinberg 2009) - aufgreifen. Das Web 2.0 scheint auch geeignet, Individuen näher aneinander zu rücken - dabei spielen Nationalgrenzen fast keine Rolle mehr (Friedman 2007). Wie Kluzer/ Rissola (2009) zeigen konnten, bestehen vor allem große Potenziale in der Überbrückung der ,digitalen Kluft' durch die Anbindung bildungsferner oder ITferner Menschen, da Web 2.0-Applikationen die technischen Hürden von medienvermittelter Kommunikation sowie IT-Nutzung stark senken.

\section{Literatur}

Alby, Tom (2007). Web 2.0. Konzepte, Anwendungen, Technologien. München: Hanser.

Back, Andrea/ Bendel, Oliver/ Stoller-Schai, Daniel (2001). E-Learning im Unternehmen. Zürich: Orell Füssli Verlag AG.

Beerheide, Emanuel/ Howaldt, Jürgen/ Kopp, Ralf (2009). Innovationsmanagement in der High-Tech-Branche. Auf dem Weg zum Unternehmen 2.0? In: BMBF-Tagungsband 2. Zukunftsforum Innovationsfähigkeit, Arbeiten - Lernen - Kompetenzen entwickeln. Bonn.

Bolz, Norbert/ Kittler, Friedrich A./ Tholen, Christoph (Hrsg.) (1994). Computer als Medium. München: Fink.

Bühl, Achim (1997). Die virtuelle Gesellschaft. Ökonomie, Politik und Kultur im Zeichen des Cyberspace. Opladen: Westdeutscher Verlag.

Dery, Mark (1996). Cyber. Die Kultur der Zukunft. Berlin: Verlag Volk \& Welt.

Flowers, Stephen/ Sinozic, Tanja/ Patel, Parimal (2009). Prevalence of User Innovation in the EU. Analysis based on the Innobarometer Surveys of 2007 and 2009. Download von: PRO INNO Europe (http://www.proinnoeurope.eu/admin/uploaded_documents/EIS_2009_User_innovation.pdf, Abruf: 26.01.2010).

Friedmann, Thomas L. (2007). Die Welt ist flach. Eine kurze Geschichte des 21. Jahrhunderts. Frankfurt a.M.: Suhrkamp.

Goertz, Lutz (1995). Wie interaktiv sind die Medien? Auf dem Weg zu einer Definition von Interaktivität. Rundfunk und Fernsehen, 21 (4), 476-493.

Groys, Boris (1999). Über das Neue. Versuch einer Kulturökonomie. Frankfurt a.M.: Hanser.

Haack, Johannes (1995). Interaktivität als Kennzeichen von Multimedia und Hypermedia. In: L. J. Issing/ P. Klimsa (Hrsg.), Information und Lernen mit Multimedia (S. 151166). Weinheim: BeltzPVU.

Heidenreich, Martin (2004). Innovationen und soziale Sicherung im internationalen Vergleich. Soziale Welt 2/2004, 125-144.

Hippel von, Eric (2005). Democratizing Innovation. Cambridge, Massachusetts, London: The MIT Press.

Howaldt, Jürgen/ Kopp, Ralf/ Schwarz, Michael (2008). Innovationen (forschend) gestalten. Zur neuen Rolle der Sozialwissenschaften. WSI-Mitteilungen 2/2008, 63-69. 
Howaldt, Jürgen/ Schwarz, Michael (2009). Soziale Innovation - Konzepte, Forschungsfelder und -perspektiven. Unveröffentlichtes Manuskript.

Höflich, Joachim R. (1995). Vom dispersen Publikum zu „elektronischen Gemeinschaften“. Plädoyer für einen erweiterten kommunikationswissenschaftlichen Blickwinkel. Rundfunk und Fernsehen 43 (4), 518-537.

Hügli, Daniel (1997). Daten und Netze. Akzeptanz, Nutzung und kommunikatives Veränderungspotential der Informations- und Kommunikationstechnologien. Bern, Berlin, Frankfurt a.M., New York, Paris, Wien: Peter Lang.

Kluzer, Stefano/ Rissola, Gabriel (2009). E-Inclusion Policies and Initiatives in Support of Employability of Migrants and Ethnic Minorities in Europe. Information technologies \& International Development, 5 (2), 67-76.

Krämer, Sybille (Hrsg.) (1998). Medien Computer Realität. Wirklichkeitsvorstellungen und Neue Medien. Frankfurt a.M.: Suhrkamp.

Kubicek, Herbert (1997). Bürgerinformation durch „neue“ Medien? Analysen und Fallstudien zur Etablierung elektronischer Informationssysteme im Alltag. Opladen: Westdeutscher Verlag.

Kuhlen, Rainer (1999). Die Konsequenzen von Informationsassistenten. Frankfurt a.M.: Suhrkamp.

Langenbucher, Wolfgang R. (1985). Der Ausbau des drucktechnischen Kommunikationssystems. Skizzen zu einem „Printkommunikationsbericht“. In: E. Schreiber/ W. R. Langenbucher/ W. Hömberg (Hrsg.), Kommunikation im Wandel der Gesellschaft. Festschrift für Otto B. Roegele (S. 269-280). Konstanz: Universitätsverlag.

Leskovec, Jure/ Backstrom, Lars/ Kleinberg, Jon (2009). Meme-tracking and the Dynamics of the News Cycle. Download von: School of Computer Science, Carnegie Mellon (http://www.cs.cmu.edu/ jure/pubs/quotes-kdd09.pdf, Abruf: 26.01.2010).

Maaß, Christian/ Pietsch, Gotthard (2007). Web 2.0 als Mythos, Symbol und Erwartung. Diskussionsbeitrag der Fakultät für Wirtschaftswissenschaft der Fernuniversität in Hagen.

Münchener Kreis e. V./ Deutsche Telekom AG/ TNS Infratest GmbH/ European Center for Information and Communication Technologies (EICT) GmbH (Hrsg.) (2008). Zukunft \& Zukunftsfähigkeit der deutschen Informations- und Kommunikationstechnologie. Abschlussbericht der ersten Projektphase. Download von: Zukunft \& Zukunftsfähigkeit der deutschen Informations- und Kommunikationstechnologie (http://www.downloadtelekom.de/dt/StaticPage/59/97/56/081205_zukunftsstudie.pdf_599756.pdf, Abruf: 26.01.2010).

Neuberger, Christoph/ Nuernbergk, Christian/ Rischke, Melanie (2007). Weblogs und Journalismus: Konkurrenz, Ergänzung oder Integration? Eine Forschungssynopse zum Wandel der Öffentlichkeit im Internet. Media Perspektiven 2/2007, 96-112.

Neverla, Irene (Hrsg.) (1998). Das Netz-Medium. Kommunikationswissenschaftliche Aspekte eines Mediums in Entwicklung. Opladen: Westdeutscher Verlag.

Pelka, Bastian (2003). Künstliche Intelligenz und Kommunikation. Delphi-Studie zur Technikfolgenabschätzung des Einflusses von KI auf Kommunikation, Medien und Gesellschaft. Münster: Lit-Verlag. 
Pelka, Bastian (Hrsg.) (2008). Das Prinzip Wiki in der Praxis: Theorie, Anwendung, Anleitung. Tönning, Lübeck, Marburg: Der Andere Verlag.

Pelka, Bastian/ Görting, Leonie/ Schmitt, Julia (2008). Potenziale von Wikis in der Hochschullehre. Eine Theorie geleitete explorative Erprobung von Konzeptionsprinzipien für die Nutzung von Wikis in der Web 2.0-gestützten Lehre. Download von: eteaching.org (http://www.e-teaching.org/materialien/praxisberichte/Pelka-Wiki, Abruf: 26.01.2010).

Pohlmann, Mark (2007). Was wird mit den Blogs? Download von: Themenblog (http://www.themenblog.de/2007/05/was_kommt_nach_blogs.html, Abruf: 26.01.2010).

Quiring, Oliver/ Schweiger, Wolfgang (2006). Interaktivität - ten years after. Bestandsaufnahme und Analyserahmen. Medien \& Kommunikationswissenschaft, 54 (1), 524.

Reißmann, Ole (2005). Was Weblogs sein können. In: Netzwerk Recherche (Hrsg.), Online-Journalismus. Chancen, Risiken und Nebenwirkungen der InternetKommunikation (S. 63-65). Wiesbaden: Verlag Netzwerk Recherche.

Rheingold, Howard (1992). Virtuelle Welten. Reisen im Cyberspace. Hamburg: Reinbek.

Tapscott, Don/ Williams, Anthony D. (2007). Wikinomics - Die Revolution im Netz. München: Hanser.

Wasserman, Stanley/ Faust, Katherine (1994). Social Network Analysis: Methods and Applications. Download von: Google books (http://books.google.de/books?hl=de\&lr=\&id=CAm2DpIqRUIC\&oi=fnd\&pg=PR21 $\underline{\text { \&dq=social+network\&ots=HtHmseYzPb\&sig=ZTts3PyApsjIJT0FQIf7fAwTo2c\#P }}$ PA27,M1, Abruf: 26.01.2010).

Weischenberg, Siegfried (1990). Nachrichtenschreiben. Westdeutscher Verlag: Opladen.

Weischenberg, Siegfried (1998). Pull, Push und Medien-Pfusch. Computerisierung kommunikationswissenschaftlich revisited. In: I. Neverla (Hrsg.), Das NetzMedium. Kommunikationswissenschaftliche Aspekte eines Mediums in Entwicklung (S. 37-61). Opladen: Westdeutscher Verlag.

Wirth, Werner/ Schweiger, Wolfgang (1999). Selektion neu betrachtet: Auswahlentscheidungen im Internet. In: W. Schweiger/ W. Wirth (Hrsg.), Selektion im Internet. Empirische Analysen zu einem Schlüsselkonzept (S. 43-74). Opladen: Westdeutscher Verlag.

Zapf, Wolfgang (1989). Über soziale Innovationen, Soziale Welt 40 (1-2), 170-183.

\section{Webseiten}

ARD/ZDF Onlinestudie 2009. Nachfrage nach Videos und Audios steigt weiter: http://www.ard.de/intern/basisdaten/onlinenutzung/-/id=55208/59yqoa/index.html (Abruf: 26.01.2010). 